\title{
Accumulation of HIV-1 drug resistance in patients on a standard thymidine analogue-based first line antiretroviral therapy after virological failure: implications for the activity of next-line regimens from a longitudinal study in Mozambique
}

Andrea De Luca ${ }^{1 *}$, Zita Jorge Sidumo², Giacomo Zanelli ${ }^{1}$, Noorjehan Abdul Magid ${ }^{2}$, Richard Luhanga ${ }^{3}$, Davide Brambilla ${ }^{4}$, Giuseppe Liotta ${ }^{5}$, Sandro Mancinelli ${ }^{5}$, Maria Cristina Marazzi ${ }^{6}$, Leonardo Palombi ${ }^{5}$ and Susanna Ceffa ${ }^{4}$

\begin{abstract}
Background: We describe the accumulation of HIV-1 drug resistance and its effect on the activity of next-line components in patients with virological failure (HIV-1 RNA >1000 copies/mL) after 1 year (t1) of first-line antiretroviral therapy (ART) not switching to second-line drugs for one additional year (t2) in low-middle income countries (LMIC).

Methods and results: We selected 48 patients from the DREAM cohort (Maputo, Mozambique); their median pre-ART CD4+ cell count was 165 cells/ $\mu$ l. At t1 patients were receiving ART since a median of 12.2 months (mainly zidovudine/ lamivudine/nevirapine), their median HIV RNA was $3.8 \log 10 \mathrm{copies} / \mathrm{mL}$, 43 (89.6\%) presented at least one resistanceassociated mutation (RAM), most frequently for lamivudine/emtricitabine, nevirapine and efavirenz. Resistance to tenofovir, was $10 \%$ at 1 year and higher than $20 \%$ at 2 years, while projection at 3 years was $>30 \%$. At t2, 42 (89.4\%) had a predicted low-level or higher resistance to at least $1 \mathrm{~s}$-line drug. At $\mathrm{t} 1$, the frequency of RAM in patients with a lower adherence to pharmacy appointments (<95\%) was significantly lower (12/20,60\% for NRTI and 14/20, 70\% for NNRTI) than in those with a better adherence $(26 / 28,92.8 \%$ for NRTI and $25 / 28,89.3 \%$ for NNRTI) (OR $0.12,95 \%$ $\mathrm{Cl} 0.02-0.63, p=0.012$ and $\mathrm{OR} 0.28,95 \% \mathrm{Cl} 0.06-1.29, p=0.103$, respectively). Overall thymidine analogue mutations (TAMs) accumulation rate was 0.32/year, 0.50/year in the subgroup with HIV RNA >10,000 copies/mL; NNRTI RAM accumulation rate was 0.15/year, 0.40/year in the subgroup with HIV RNA >10,000 copies/mL.
\end{abstract}

Conclusions: While the activity of NNRTIs is compromised early during failure, tenofovir and zidovudine activity are reduced more frequently after 1 year of documented virological failure of thymidine analogue-based first-line ART, with RAMs accumulating faster in patients with higher viral loads. The present observation may help informing decisions on when to switch to a second line ART in patients on virological failure in LMIC.

Keywords: HIV-1, Antiretroviral drug resistance, Second-line ART, Virological failure, HIV-1 RNA

\footnotetext{
* Correspondence: andrea.deluca@unisi.it

${ }^{1}$ UOC Malattie Infettive Universitarie, AOU Senese and Department of

Medical Biotechnologies, Siena University Hospital, Viale Bracci 16, 53100

Siena, Italy

Full list of author information is available at the end of the article
} 


\section{Background}

As of December 2016, 19,5 million people were receiving antiretroviral therapy (ART) word-wide. As antiretroviral treatment is rapidly being scaled up, HIV drug resistance is emerging and increasing at the global level. WHO recently reported that levels of pre-treatment drug resistance (PDR) to nevirapine and efavirenz, the two most affordable non-nucleoside reverse transcriptase inhibitors (NNRTIs) used in the first-line ART in resource-limited settings, was $10 \%$ or above in 6 of 11 countries performing nationally representative HIV drug resistance (DR) surveys, 3 out of 4 in Africa [1]. According to a systematic review, the estimated annual incremental rate of PDR to NNRTIs ranged between $15 \%$ and $29 \%$ in Africa [2]. In addition, NNRTI resistance among people with unsuppressed viral load on first-line NNRTI regimens ranged from $47 \%$ to $90 \%$. A systematic review of studies of acquired drug resistance (ADR) in adults from 30 lowmiddle income countries published between 2014 and 2016 found that $68 \%$ of those failing NNRTI regimens had one or more resistance mutation after a median time on ART of 15 months. Resistance was more frequent for NNRTI (61\%), as for nucleoside/nucleotide reverse transcriptase inhibitors (NRTI) (55\%) [1]. Viral load monitoring is recommended by WHO in order to diagnose and confirm ART failure and provide an early and more accurate indication to switch treatment from first-line to secondline drugs, reducing the accumulation of resistance mutations [3]. Lower viral load is associated with less HIV-1 resistance mutations to all drug classes and viral load monitoring is expected to significantly reduce the emergence of acquired HIV drug resistance [3-8]. WHO consolidated HIV guidelines recommend a NNRTIs-based regimen as first line ART [3]. After failure patients may be switched to boosted protease-inhibitors (PIs)-based second-line ART [3]. However, given the paucity of next treatment lines in low-middle income countries (LMIC), the correct switching time should, among others, be informed by the probability of accumulating resistance to the NRTI agents included in the second line at a given time of virological failure [3, 6-9]. Objectives of this study are to describe the accumulation of HIV-1 drug resistance and its effect on the activity of next-line therapy components in patients undergoing viral load monitoring and showing virological failure after 1 year of first-line ART but not switching to second-line ART for one additional year.

\section{Methods}

A retrospective longitudinal cohort study was performed with parameters extracted from the Drug Resource Enhancement against AIDS and Malnutrition (DREAM) database for HIV-infected patients. DREAM is a program of care for people with HIV designed and managed by the Community of Sant'Egidio (Italy), which is now working in ten African countries including Mozambique $[3,10]$. The DREAM program offers free of charge stateof-the-art treatment, diagnostic facilities and nutritional supplementation to all HIV- infected patients within centers from the public sector [3, 10]. Antiretroviral therapy is supplemented by the governmental program following National guidelines. Patients on ART perform plasma viral load monitoring at yearly intervals. We selected patients from 3 sites in Maputo (Mozambique) who a) were on a first-line ART b) had an HIV RNA $>1000$ copies/mL after 1 year $(\mathbf{t} \mathbf{1})$ and after 2 years $(\mathbf{t} 2)$ of ART without switching to a second-line regimen and c) had a stored plasma sample at $\mathrm{t} 1$ and $\mathrm{t} 2$. We also considered: $\mathbf{t} \mathbf{2}^{\wedge}$ : 2 years after ART initiation (1 year after first documented virological failure) considering historical genotype; $\mathbf{t 3}^{*}$ : 3 years after ART initiation (2 years after first documented virological failure), projected results assuming a continuous linear accumulation of resistance mutations and historical genotype at year 2 (cumulating resistance of $\mathrm{t} 1+\mathrm{t} 2$ ). Genotyping of reverse transcriptase (RT) and protease was performed at $\mathrm{t} 1$ and $\mathrm{t} 2$ by the Trugene HIV-1 genotyping method (Siemens Health Care Diagnostics, NY, USA). Proportion of subjects with major resistance associated mutations (RAM) (International Antiviral Society-USA list 2014) to NRTIs, thymidine analogue mutations (TAMs) and NNRTI RAM and with resistance to individual drugs as interpreted by Stanford's HIVdb 7.0 system (considering at least low level resistance, LLR to each drug) was calculated. Adherence was measured by per cent on time pharmacy appointment keeping, allowing a delay tolerability of 10 days. The protocol was approved by the National Ethics Committee from Mozambique (approval n. 384/CNBS/12 on October 18, 2012).

\section{Statistical analysis}

Baseline characteristics of study patients were described using standard descriptive statistics. Associations between socio-demographic and HIV-related variables were assessed using the Chi-square test for categorical variables and Student's t-test or the Mann - Whitney test for continuous variables, as required. Association between adherence to pharmacy appointments and resistance mutations was analyzed by logistic regression. Data were analyzed using SPSS software package (version 18.0 Chicago, IL).

\section{Results}

There were 48 eligible patients enrolled in the study: 24 (50\%) were males. At baseline (time of ART initiation, median calendar year 2010) the median patients's age was 35 years (inter-quartile range, IQR 28.5-37.7), the median CD4+ cell count was 165 cells/ $\mu l$, and the mean viral load was $4.69 \log _{10}$ copies/ml. Most of the patients (95.8\%) were infected with HIV-1 subtype C. At t1, 
patients were receiving ART for a median of 12.2 months and the most frequently employed regimen was zidovudine/lamivudine/nevirapine $(n=36,75 \%)$, followed by stavudine/lamivudine/nevirapine $(n=4,8.3 \%)$, stavudine/ lamivudine/efavirenz $(n=3,6.2 \%)$, zidovudine/lamivudine/abacavir $(n=3,6.2 \%)$ and zidovudine/lamivudine/ efavirenz $(n=2,4.2 \%)$. Between $\mathrm{t} 1$ and t2, NRTI components were substituted in 2 cases from zidovudine/lamivudine to stavudine/lamivudine, in 2 cases from stavudine/ lamivudine to zidovudine/lamivudine and in 2 cases from stavudine/lamivudine to tenofovir/lamivudine. Table 1 shows the main clinical and virological characteristics of the patients at time t1 and t2. Out of 48 patients, 43 (89.6\%) presented at least one RAM after one year of ART and 34/48 (70.8\%), showed at least one RAM both for NRTI and NNRTI. The proportion of RAM showed an increase from t1 to t2 (Fig. 1a). In relation to individual drugs the most frequent resistance mutations were observed for lamivudine and emtricitabine, nevirapine and efavirenz with progressive accumulation over time (Fig. 1b). Resistance to tenofovir, which is the cornerstone drug of the NRTI backbone therapy for the second-line regimen in patients failing a first-line regimen containing thymidine-analogues was $10 \%$ at 1 year and slightly higher than $20 \%$ at 2 years, when cumulative resistance was considered; projection at 3 years showed a $>30 \%$ probability of tenofovir resistance. Two of 47 (4.3\%) patients with a protease sequence available had major PI resistance mutations (I54T and L90 M each in one patient), conferring LLR to atazanavir/r and lopinavir/r. We then considered the predicted resistance to the candidate second-line regimens based on WHO recommendations and the type of first-line regimen failing. At t1 31 (64.6\%) had at least LLR to 1 second-line drug and another 5 (10.4\%) to 2 drugs, so that a total of $36(75 \%)$ had at least 1 second-line drug with predicted low-level or higher resistance. At t2 31 (66.0\%) had at least LLR to 1 second-line drug and another $11(23.4 \%)$ to 2 drugs, so that a total of $42(89.4 \%)$ had at least 1 second-line drug with predicted low-level or higher resistance.

Table 1 Clinical and virological follow-up at t1 ( $n=48$ patients) and 2 ( $n=47$ patients)

\begin{tabular}{|c|c|c|}
\hline & t1 & t2 \\
\hline 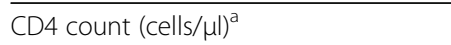 & $234(113-322)$ & $236(136-386)$ \\
\hline HIV RNA ( $\log _{10}$ copies/ml) & $3.8(3.5-4)$ & $3.9(3,5-4.5)$ \\
\hline $\mathrm{Hb}(\mathrm{g} / \mathrm{dL})^{\mathrm{a}}$ & $12.6(11.3-13.1)$ & $12.1(11.2-13.3)$ \\
\hline BMI $\left(K g / m^{2}\right)^{a}$ & $22.2(20.7-25.3)$ & $21.9(20.5-24.7)$ \\
\hline Adherence (\%) ${ }^{a}$ & $100(92-100)$ & $100(92-100)$ \\
\hline $\begin{array}{l}\text { Percent of patients with }<95 \% \\
\text { adherence to pharmacy appointments }\end{array}$ & 41.7 & 41.3 \\
\hline
\end{tabular}

At $\mathrm{t} 1$, the probability of RAM in patients on virological failure differed based on levels of adherence to pharmacy appointments. In particular, frequency of NRTI RAM in patients with a lower adherence to pharmacy appointments $(<95 \%)$ was significantly lower $(12 / 20,60 \%)$ than in those with a better adherence $(26 / 28,92.8 \%)$ (OR 0.12, $95 \%$ CI $0.02-0.63, p=0.012$ ). The mean yearly TAMs accumulation rate was 0.32 ; which rose to 0.49 in patients with pharmacy appointment adherence $>90 \%$. In patients with HIV-1 RNA > 10,000 copies/mL TAMs showed a mean yearly accumulation rate of 0.50 . Probability of NNRTI resistance was also lower in less adherent patients at t1 (14/20, 70\% vs $25 / 28,89.28 \%$; OR 0.28 , 95\% CI $0.06-$ $1.29, p=0.103)$. Mean NNRTI RAMs accumulation rate was $0.15 /$ year. In subjects with pharmacy appointment adherence $>90 \%$ the mean accumulation rate was $0.17 /$ year. In patients with HIV-1 RNA > 10,000 copies/mL the mean yearly accumulation rate of NNRTI RAMs was 0.40.

\section{Discussion}

Mozambique, a sub-Saharan country with HIV prevalence of $10.6 \%$ provides antiretroviral therapy based on a public health approach $[3,11,12]$. Treatment options for HIV infected people in low-middle income countries (LMIC) are based on WHO guidelines [3]. First-line regimen includes two NRTI (tenofovir or zidovudine and lamivudine or emtricitabine) plus one NNRTI (nevirapine or efavirenz) whereas a boosted protease inhibitors-based ART is used as second line regimen with a substitution of the NRTIs: tenofovir is given after zidovudine failure, while zidovudine is recommended in the second-line after tenofovir has failed in the first-line regimen [3].This treatment sequencing strategy is based on the rationale that after virological failure with 2 NRTI + a NNRTI, the activity of the protease inhibitors is preserved and the crossresistance of the alternate NRTIs is limited $[3,6,13]$. However, after tenofovir failure, HIV-1 usually selects for RAMs such as K65R that do not affect zidovudine activity; on the contrary zidovudine selects for TAMs that accumulate and progressively confer increasing cross-resistance to tenofovir. This is one of the reasons why WHO now recommends to prefer tenofovir as first-line regimen, but many countries still use zidovudine due to cost and procurement issues. Viral load monitoring to detect treatment failures, is now recommended by WHO and, although not yet available in many areas, is becoming increasingly accessible [3]. Consideration of the timing of treatment switch after 1st-line ART failure is particularly important in resource-limited settings where salvage regimens are scarce and costly [9]. For these reasons in LMIC, the correct switching time should also be informed by the probability of accumulating resistance to the subsequent treatment lines at a given time of virological failure $[7,9,13]$. In this study, we report how RAM 

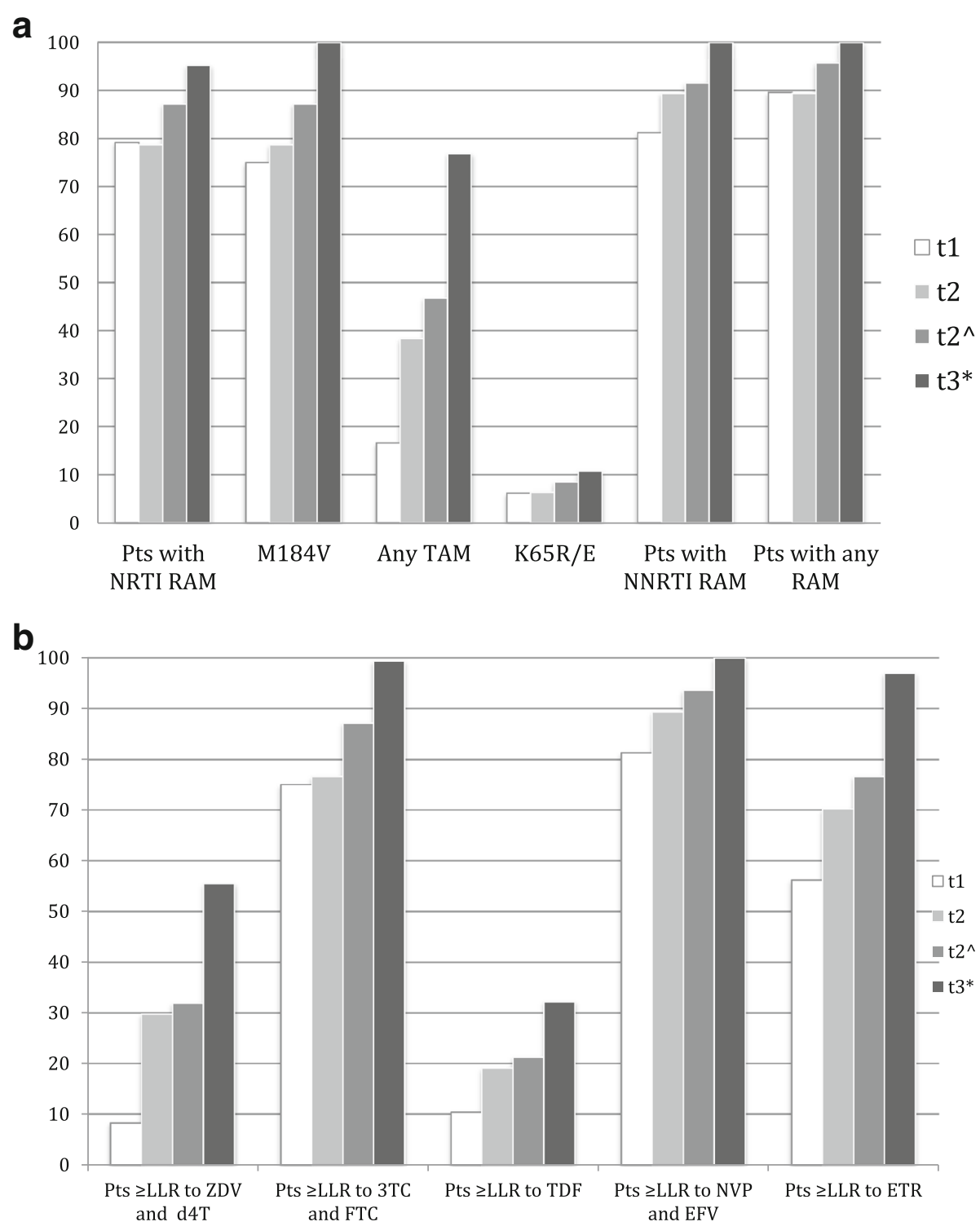

Fig. 1 a Proportion of patients with major resistance mutations at $\mathrm{t} 1^{*}$ (first documented HIV RNA $>1000$ copies/mL at 1 year of ART) and t2 *(continued HIV RNA $>1000$ copies $/ \mathrm{mL}$ at 2 years of ART). t2^ represents the cumulative resistance summing resistance at t 1 and t2. t 3 represents the projection of resistance at 3 years based on $\mathrm{t} 1$ and $\mathrm{t} 2 \wedge$ and assuming absence of resistance at baseline. $\mathbf{b}$ Proportion of patients with predicted resistance to individual drugs at $\mathrm{t} 1$ and $\mathrm{t} 2$ and the projection at 3 years. ${ }^{*} \mathrm{t} 1$ total patients $n=48$, t2 total patients $n=47$; NRTI, nucleoside/nucleotide reverse-transcriptase inhibitor; NNRTI, non-nucleoside reverse-transcriptase inhibitor; Pls, protease inhibitors; RAM, resistance-associated mutations; TAM thymidine analog mutation; LLR, low-level resistance according to the interpretation by hivdb v 7.0; ZDV, zidovudine; 3TC, lamivudine; TDF, tenofovir; NVP, nevirapine; EFV, efavirenz; ETR, etravirine

accumulate after virological failure of a thymidine analogue-based first-line regimen in a resource-limited setting. In particular, we show that cross-resistance to tenofovir was still limited when failure was detected at 1 year after ART initiation. After 2 years and, in projection, after 3 years, cross-resistance to tenofovir accumulated significantly. This lead to a significant accumulation of resistance to drugs that, based on WHO guidelines, would have been used for the second-line regimen: a predicted low-level or higher resistance to at least 1 drug of the second-line regimen rose from $75 \%$ of cases at t1 to $89 \%$ at $\mathrm{t} 2$, while $10 \%$ at $\mathrm{t} 1$ and $23 \%$ at $\mathrm{t} 2$ showed a predicted resistance to 2 second-line drugs. Previous studies have anayzed the accumulation of drug resistance mutations in patients failing first-line regimens in Sub-Saharan Africa. In a retrospective study in South Africa, in 43 patients performing sequential resistance tests with a median interval of 5 months, RAMs accumulated at a mean of $0.07 /$ month of drug exposure [14]. In a prospective cohort of Zambian children on first-line ART, 6 had sequential 
genotypes while failing on stavudine/lamivudine/nevirapine and showed an accumulation rate of 0.59 TAMs/ year [15]. In a retrospective analysis of a randomized study performed in African countries on 36 genotype pairs from weeks 48 and 96 of first-line ART, the mean TAMs accumulation rate was 1.50 /year in nevirapinetreated participants and 1.82/year in abacavir-treated participants [16]. In a retrospective analysis of adults and children failing NNRTI-based first-line ART, NNRTI resistance mutations accumulated at 0.62 /year and NRTI resistance mutations at $0.84 /$ year [17]. In our study we observed a mean TAM accumulation rate of 0.32 /year; which rose to 0.49 /year in patients with a pharmacy refill adherence $>90 \%$. The yearly accumulation rate of NNRTI resistance mutations was 0.15 , rising at 0.17 in adherent patients. An hypothesis for the reason for the lower rate of resistance accumulation in this cohort as compared to previous reports may be the availability of viral load monitoring. Indeed, patients included here were selected among those not switching to second-line for one year despite documented virologic failure. The population included showed relatively low viral loads at failure, and this might have been a reason for keeping them on first-line, prompting adherence interventions before switching to second-line. This probably selected a population at lower risk of resistance accumulation, as reported by other studies relating drug resistance accumulation to viral load $[4$, 18]. In agreement with this, patients with an HIV-1 RNA $>10,000$ copies $/ \mathrm{mL}$ in this study showed higher yearly accumulation rates for TAMs and NNRTI resistance mutations ( 0.50 and 0.40 , respectively), values that are closer to those provided by previous reports. Our findings may represent a practical indication for the management of patients with virological failure in this settings. In particular, after initial detection of virological failure patients may still benefit from adherence counselling strategies without major risk of accumulating significant cross-resistance to second-line drugs. However, the risk of resistance accumulation is higher in patients with an HIV-1 RNA above 10,000 copies $/ \mathrm{ml}$, and if virological failure persists subsequently, despite adherence implementation, a switch to second-line ART should be recommended, as RAM will accumulate in the majority of patients. Our findings should be interpreted with caution given the limited sample size and the retrospective design of the study and require validation in a prospective study. Moreover, our sequencing technique did not cover the mutation N348I in the connection domain, which is frequently selected by nevirapine and thymidine analogues in subtype $C$, and slightly reduces susceptibility to NNRTIs and zidovudine, so our analyses may have slightly underestimated resistance to these agents [19]. However, the accurate patients selection and follow up and the contemporary longitudinal assessment of viral load, resistance and adherence at specified times on ART represent strong points of this cohort study. It is important to consider that studies in SubSaharan Africa have shown that viral load could be successfully re-suppressed with boosted PI-based second-line regimens despite the presence of NRTI resistance after first-line failure. One observational cohort study showed successful virologic suppression with second-line drugs, despite $53 \%$ were predicted to receive partially active regimens due to drug resistance [20]; moreoever, in a randomized clinical trial [21] response to a second-line regimen based on boosted PI +2-3 NRTIs was better as compared to a boosted PI + raltegravir or boosted PI monotherapy despite NRTIs had no predicted activity due to resistance mutations; finally, in another randomized trial, the major determinant of response to boosted PI $+2-3$ NRTIs as second-line regimen was adherence but not baseline resistance [22]. Therefore, our findings on NRTI RAM accumulation on first-line failing patients may have limited implications for clinical practice given the residual activity of a regimen based on boosted PI + NRTIs. It remains to be established whether the introduction of dolutegravir in the recommended first-line or second-line regimens will change this scenario.

\section{Conclusions}

While the activity of NNRTIs is compromised early during failure, tenofovir and zidovudine activity begin to be reduced more frequently after 1 year of documented virological failure of thymidine analogue-based first-line ART. Higher viral load at failure is associated with a faster rate of resistance accumulation. The present observation may inform decisions on when to switch to a second line therapy in patients on virological failure in LMIC settings.

\section{Abbreviations \\ 3TC: Lamivudine; ADR: Acquired drug resistance; ART: Antiretroviral therapy; DREAM: Drug Resource Enhancement against AIDS and Malnutrition; EFV: Efavirenz; ETR: Etravirine; HIV: Human immunodeficiency virus; LLR: Low-level resistance; LMIC: Low-medium income countries; NNRTI: Non- nucleoside reverse-transcriptase inhibitor; NRTI: Nucleoside/nucleotide reverse-transcriptase inhibitor; NVP: Nevirapine; PDR: Pre-treatment drug resistance; Pls: Protease inhibitors; RAM: Resistance-associated mutations; TAM: Thymidine analog mutation; TDF: Tenofovir; WHO: World Health Organization; ZDV: Zidovudine}

\section{Acknowledgements}

None to declare.

\section{Funding}

The DREAM program supplied all reagents, materials and structures in the interest of its patients. No specific funding has been provided during the study.

Availability of data and materials

The data and materials are available from the corresponding author on reasonable request (Andrea De Luca; Dept. Medical Biotechnology, Siena University Hospital; email: andrea.deluca@unisi.it). 


\section{Authors' contributions}

ADL and SC designed the study. ZJS, NAM, RL, DB, GL, SM, MCM, LP, SC conceived the study, participated in the design of the study, collection of data, and drafting and revision of the manuscript. ADL, GZ carried out the statistical analyses reported, interpreted the results and drafted, revised and finalised the manuscript. All authors read and approved the final manuscript.

\section{Ethics approval and consent to participate}

The protocol was approved by the National Ethics Committee from Mozambique (approval n. 384/CNBS/12 on October 18, 2012). All patients gave a written informed consent to participate.

\section{Consent for publication}

Not applicable.

\section{Competing interests}

ADL is a Section Editor of BMC Infectious Diseases.

\section{Publisher's Note}

Springer Nature remains neutral with regard to jurisdictional claims in published maps and institutional affiliations.

\begin{abstract}
Author details
'UOC Malattie Infettive Universitarie, AOU Senese and Department of Medical Biotechnologies, Siena University Hospital, Viale Bracci 16, 53100 Siena, Italy. ${ }^{2}$ DREAM Program, Maputo, Mozambique. ${ }^{3}$ DREAM Program, Malawi and UPO (Università del Piemonte Orientale), Novara, Italy. ${ }^{4}$ DREAM Program, Rome, Italy. ${ }^{5}$ nniversity of Tor Vergata, Rome, Italy. ${ }^{6}$ University LUMSA, Rome, Italy.
\end{abstract}

Received: 26 March 2017 Accepted: 29 August 2017

Published online: 05 September 2017

\section{References}

1. World Health Organization. HIV drug resistance report 2017. Geneva: World Health Organization. 2017. Licence: CC BY-NC-SA 3.0 IGO.

2. Mbuagbaw L, Aves T, Borhan S, Leenus A, Jordan MJ, Parkin N, et al. Systematic reviews and meta-analyses used to inform the World health Organization Guidelines on the public health response to pre-treatment HIV drug resistance. 2017. [Publication pending]. Web Annex 2. In: Guidelines on the public health response to pre-treatment HIV drug resistance. Geneva: World health Organization; 2017. available at http://who.int/hiv/pub/ guidelines/hivdr-guidelines-2017/

3. WHO. Consolidated guidelines on the use of antiretroviral drugs for treating and prevent HIV infection: recommendations for a public health approach. In: Second edition; 2016. http://apps.who.int/iris/bitstream/10665/208825/1/ 9789241549684_eng.pdf?ua=1.

4. Prosperi MC, Mackie N, Di Giambenedetto S, Zazzi M, Camacho R, Fanti I, et al. Detection of drug resistance mutations at low plasma HIV-1 RNA load in a European multicentre cohort study. J Antimicrob Chemother. 2011;66:1886-96.

5. Pham QD, Wilson DP, Nguyen TV, Do NT, Truong LX, Nguyen LT, et al. Projecting the epidemiological effect, cost-effectiveness and transmission of HIV drug resistance in Vietnam associated with viral load monitoring strategies. J Antimicrob Chemother. 2016;71:1367-79

6. De Luca A, Marazzi MC, Mancinelli S, Ceffa S, Altan AM, Buonomo E, et al. Prognostic value of virological and immunological responses after 6 months of antiretroviral treatment in adults with HIV-1 infection in sub-Saharan Africa. J Acquir Immune Defic Syndr. 2012;59:236-44.

7. Rawizza HE, Chaplin B, Meloni ST, Eisen G, Rao T, Sankalè JL, et al. Immunologic criteria are poor predictors of virologic outcome: implications for HIV treatment monitoring in resource-limited settings. Clin Infect Dis. 2011:53:1283-90

8. Sigaloff $\mathrm{KC}$, Hamers RL, Wallis $\mathrm{CL}$, Kityo $\mathrm{C}$, Siwale $\mathrm{M}$, Ive $\mathrm{P}$, et al. Unnecessary antiretroviral treatment switches and accumulation of HIV resistance mutations; two arguments for viral load monitoring in Africa. J Acquir Immune Defic Syndr. 2011;58:23-31.

9. Ruperez M, Pou C, Macaluve S, Cedeno S, Luis L, Rodriguez J, et al. Determinants of virological failure and antiretroviral drug resistance in Mozambique. J Antimicrob Chemother. 2015;70:2639-47.
10. DREAM Community of Sant'Egidio. The goal of a dream. Achieving millenium development goals. http://dream.santegidio.org/wp-content/ uploads/2015/01/DREAM_MDG_def.pdf.

11. UNAIDS.HIV and AIDS estimates. 2015. http://www.unaids.org/en/ regionscountries/countries/mozambique/.

12. Bila DCA, Boullosa LT, Vubli AS, Mabunda NJ, Abreu CM, Ismael N, et al. Trends in prevalence of HIV-1 drug resistance in a public clinic in Maputo, Mozambique. PLoS One. 2015;10:e0130580. doi:10.1371/journal.pone.0130580.

13. De Luca A, Hamers RL, Shapiro JM. Antiretroviral treatment sequencing strategies to overcome HIV type 1 drug resistance in adolescents and adults in low-middle-income countries. J Infect Dis. 2013;207:563-9.

14. Sigaloff KC, Ramatsebe T, Viana R, de Wilt TF, Wallis CL, Stevens WS Accumulation of HIV drug resistance mutations in patients failing first-line antiretroviral treatment in South Africa. AIDS Res Hum Retrovirus. 2012;28:171-5.

15. Gupta RK, Ford D, Mulenga V, Walker AS, Kabamba D, Kalumbi M, et al. Drug resistance in human immunodeficiency virus type-1 infected Zambian children using adult fixed dose combination stavudine, lamivudine, and nevirapine. Pediatr Infect Dis J. 2010;29:e57-62.

16. Goodall RL, Dunn DT, Nkurunziza P, Mugarura L, Pattery T, Munderi $P$, et al. Rapid accumulation of HIV-1 thymidine analogue mutations and phenotypic impact following prolonged viral failure on zidovudine-based first-line ART in sub-Saharan Africa. J Antimicrob Chemother. 2017:72:1450-5.

17. Boender TS, Kityo CM, Boerma RS, Hamers RL, Ondoa P, Wellington M, et al. Accumulation of HIV-1 drug resistance after continued virological failure on first-line ART in adults and children in sub-Saharan Africa. J Antimicrob Chemother. 2016:71:2918-27.

18. Guichet E, Aghokeng A, Serrano L, Bado G, Toure-Kane C, Eymard-Duvernay $\mathrm{S}$, et al. Short Communication: High viral load and multidrug resistance due to late switch to second-line regimens could be a major obstacle to reach the 90-90-90 UNAIDS objectives in sub-Saharan Africa. AIDS Res Hum Retrovir. 2016:32:1159-62.

19. Brehm JH, Koontz DL, Wallis CL, Shutt KA, Sanne I, Wood R, et al. Frequent emergence of N348I in HIV-1 subtype $C$ reverse transcriptase with failure of initial therapy reduces susceptibility to reverse-transcriptase inhibitors. Clin Infect Dis. 2012;55:737-45.

20. Sigaloff KC, Hamers RL, Wallis CL, Kityo C, Siwale M, Ive P, et al. Second-line antiretroviral treatment successfully resuppresses drug-resistant HIV-1 after first-line failure: prospective cohort in sub-Saharan Africa. J Infect Dis. 2012; 11:1739-44

21. Paton NI, Kityo C, Thompson J, Nankya I, Bagenda L, Hoppe A, et al. Nucleoside reverse-transcriptase inhibitor cross-resistance and outcomes from second-line antiretroviral therapy in the public health approach: an observational analysis within the randomised, open-label, EARNEST trial. Lancet HIV. 2017:4:e341-8

22. Boyd MA, Moore CL, Molina JM, Wood R, Madero JS, Wolff M, et al. Baseline HIV-1 resistance, virological outcomes, and emergent resistance in the SECOND-LINE trial: an exploratory analysys. Lancet HIV. 2015;2:e42-51.

\section{Submit your next manuscript to BioMed Central} and we will help you at every step:

- We accept pre-submission inquiries

- Our selector tool helps you to find the most relevant journal

- We provide round the clock customer support

- Convenient online submission

- Thorough peer review

- Inclusion in PubMed and all major indexing services

- Maximum visibility for your research

Submit your manuscript at www.biomedcentral.com/submit
Biomed Central 